\title{
Padronização de medicamentos e seu impacto na assistência farmacêutica hospitalar e nos custos dos medicamentos
}

\author{
Standardization of medicines and their impact on hospital pharmaceutical care and drug costs \\ Estandarización de medicamentos y su impacto en la atención farmacéutica hospitalaria y los \\ costos de los medicamentos
}

Recebido: 28/09/2021 | Revisado: 24/10/2021 | Aceito: 25/10/2021 | Publicado: 27/10/2021

\author{
Guilherme Benigno Magalhães Duarte \\ ORCID: https://orcid.org/0000-0001-6786-0409 \\ Faculdade Integrada Carajás, Brasil \\ E-mail: guilhermebenigno15@gmail.com \\ Yolanda de Jesus Morais \\ ORCID: https://orcid.org/0000-0001-7105-8267 \\ Faculdade Integrada Carajás, Brasil \\ E-mail: yolandamorais123@gmail.com
}

\begin{abstract}
Resumo
Introdução: A Farmácia Hospitalar (FH) é uma unidade clínica, administrativa e econômica, dirigida por farmacêutico, ligada hierarquicamente à direção do hospital ou serviço de saúde e integrada funcionalmente com as demais unidades administrativas e de assistência ao paciente. Assim, a farmácia hospitalar deve desenvolver atividades clínicas e relacionadas à gestão. Metodologia: Trata-se de uma pesquisa de campo de caráter descritivo e exploratório e de abordagem qualitativa que foi realizada na farmácia hospitalar do hospital regional de Conceição do Araguaia (HRCA) na qual houve uma entrevista direcionada ao farmacêutico responsável técnico utilizando um questionário com questões que visam alcançar os objetivos deste trabalho. Resultados e discussão: As respostas dadas pelo farmacêutico na entrevista foram confrontadas com dados encontrados na literatura disponível sobre o tema, para isso foram utilizados dados de artigos publicados nos últimos 5 anos, isto é, do ano de 2017 a 2021 . Considerações finais: A FH é um setor que demanda autos valores orçamentários fazendo com que pessoas responsáveis pela instituição adotem métodos que garantam maior controle sobre as contas do hospital. A CFT é uma junta multiprofissional responsável pela seleção, padronização e outras questões relacionadas a medicamentos e tem sua importância no processo de padronização de medicamentos. Apesar do HRCA não dispor de uma CFT para executar a padronização de medicamentos ainda assim é possível realizar essa atividade sem a necessidade de uma comissão.
\end{abstract}

Palavras-chave: Farmácia hospitalar; Padronização de medicamentos; Farmacoeconomia.

\begin{abstract}
Introduction: The Hospital Pharmacy (FH) is a clinical, administrative and economic unit, run by a pharmacist, hierarchically linked to the direction of the hospital or health service and functionally integrated with the other administrative and patient care units. Thus, the hospital pharmacy must develop clinical and management-related activities. Methodology: This is a descriptive and exploratory field research with a qualitative approach that was carried out in the hospital pharmacy of the regional hospital of Conceição do Araguaia (HRCA) in which there was an interview directed to the technical responsible pharmacist using a questionnaire with questions that aim to achieve the objectives of this work. Results and discussion: The answers given by the pharmacist in the interview were compared with data found in the available literature on the subject, for this, data from articles published in the last 5 years, that is, from 2017 to 2021, were used. Final considerations: A FH is a sector that demands self-budgetary amounts, making people responsible for the institution adopt methods that ensure greater control over the hospital's bills. The CFT is a multi-professional board responsible for selection, standardization and other issues related to drugs and is important in the drug standardization process. Although the HRCA does not have a CFT to carry out the standardization of medicines, it is still possible to carry out this activity without the need for a commission.
\end{abstract}

Keywords: Hospital pharmacy; Standardization of medications; Pharmacoeconomics.

\section{Resumen}

Introducción: La Farmacia Hospitalaria $(\mathrm{FH})$ es una unidad clínica, administrativa y económica, dirigida por un farmacéutico, vinculada jerárquicamente a la dirección del hospital o servicio de salud y funcionalmente integrada con el resto de unidades administrativas y asistenciales. Por tanto, la farmacia hospitalaria debe desarrollar actividades clínicas y de gestión. Metodología: Se trata de una investigación de campo descriptiva y exploratoria con enfoque cualitativo que se llevó a cabo en la farmacia hospitalaria del hospital regional de Conceição do Araguaia (HRCA) en 
la que se realizó una entrevista dirigida al farmacéutico técnico responsable mediante un cuestionario con preguntas. que pretenden alcanzar los objetivos de este trabajo. Resultados y discusión: Las respuestas dadas por el farmacéutico en la entrevista se compararon con los datos encontrados en la literatura disponible sobre el tema, para ello se utilizaron datos de artículos publicados en los últimos 5 años, es decir, de 2017 a 2021 . Consideraciones finales: Una HF es un sector que demanda montos autofinanciados, haciendo que los responsables de la institución adopten métodos que aseguren un mayor control sobre las facturas del hospital. El CFT es una junta multiprofesional responsable de la selección, estandarización y otros temas relacionados con los medicamentos y es importante en el proceso de estandarización de medicamentos. Aunque la HRCA no cuenta con un CFT para llevar a cabo la estandarización de medicamentos, aún es posible realizar esta actividad sin necesidad de comisión.

Palabras clave: Farmacia hospitalaria; Estandarización de medicamentos; Farmacoeconomía.

\section{Introdução}

A Farmácia Hospitalar (FH) é uma unidade clínica, administrativa e econômica, dirigida por farmacêutico, ligada hierarquicamente à direção do hospital ou serviço de saúde e integrada funcionalmente com as demais unidades administrativas e de assistência ao paciente. Assim, a farmácia hospitalar deve desenvolver atividades clínicas e relacionadas à gestão (Brasil, 2019).

Espera-se que a FH desenvolva atividades clínicas relacionadas à gestão e administração de medicamentos, e estas devem ser organizadas de acordo com as características e o nível de complexidade do hospital. Dentre essas funções, sob o ponto de vista da assistência farmacêutica, compreendem a seleção, programação, aquisição, manipulação, armazenamento, distribuição e dispensação de medicamentos, além do acompanhamento e orientação aos pacientes (Lima; Souza \& Oliveira, 2020).

A comissão de farmácia e terapêutica é uma junta deliberativa, preferencialmente designada pela diretoria clínica do hospital, com a finalidade de regulamentar a padronização de medicamentos utilizados no hospital. O objetivo é padronizar medicamentos de valor terapêutico comprovado. Para tanto é necessário um documento formal para inclusão de medicamentos, apresentar estudos clínicos e outros dados para usar como parâmetro para qualificação de fornecedores (Maia Neto, 2016).

A Padronização de Medicamentos (PM) na área de FH busca selecionar fármacos que abrangem as necessidades terapêuticas dos indivíduos que se deseja atender, considerando as peculiaridades e características de cada um. Além disso, colabora nos processos de aquisição, armazenamento, distribuição e gerenciamento do estoque, pois racionaliza a quantidade de produtos em estoque (Viana \& Nunes, 2019).

Existe uma infinidade de medicamentos disponíveis pela indústria farmacêutica, em concentração e formas diferentes, o que dificulta a escolha do mais adequado e pode ocasionar a aquisição de produtos com a mesma finalidade, resultando em desperdícios para a instituição. Por isso a importância da organização de uma comissão de farmácia e terapêutica para escolha correta dos medicamentos que serão padronizados no hospital, essa comissão deve ser composta tanto por farmacêuticos quanto por médicos, enfermeiros e outros profissionais de saúde (Lima; Souza \& Oliveira, 2020).

Os medicamentos são de fundamental importância na prevenção e recuperação da saúde e contribuem para uma melhor qualidade de vida. Porém, a utilização inadequada dos medicamentos constitui uma das principais complicações à saúde e de prejuízos sociais e econômicos. O uso de medicamentos é um exemplo de sistema complexo que exige prescrição adequada de um regime terapêutico, dispensação, preparo e técnica de administração corretos (Aizenstein \& Tomassi, 2011).

A FH é um setor que demanda altos valores orçamentários e que exige do farmacêutico capacidade para realizar atividades gerenciais e que garantam a eficiência administrativa (Viana \& Nunes, 2019). Os custos dos medicamentos devem ser considerados durante todo processo de administração dos medicamentos. Alguns fármacos necessitam de manipulações mais complexas ou uso de correspondentes específicos, ampliando o custo final de seu uso (Silva, 2020).

A padronização de medicamentos pode ser entendida como uma relação de medicamentos básicos que devem constituir os estoques das farmácias hospitalares (Maia Neto, 2016). Essa relação visa o uso racional através da seleção de 
produtos com eficácia e segurança cientificamente comprovadas, redução da quantidade de medicamentos em estoque, controle e agilidade na dispensação e maior eficiência administrativa na alocação de recursos para aquisição de insumos farmacêuticos (Nascimento; Vieira \& Senhorin, 2020). Além disso existe um enorme número de fármacos da mesma classe terapêutica disponíveis no mercado, a padronização desses fármacos uniformiza a terapêutica, diminui a quantidade de medicamentos vencidos e reduz os seus custos (Mendes; Silva \& Cavalcanti, 2015).

Por isso é necessário que haja um estudo mais amplo e aprofundado sobre a padronização de medicamentos na farmácia hospitalar. Devido à grande variedade de medicamentos disponíveis no mercado com princípios ativos em diferentes quantidades e associado a diferentes excipientes em diferentes formas farmacêuticas, fazer uma relação de medicamentos fundamentais a serem utilizados por todos os setores do hospital é muito importante, pois ajuda a reduzir problemas relacionados aos medicamentos, seja com reações adversas, ou mesmo com custos elevados de aquisição dos medicamentos.

Com base nas pesquisas em artigos, livros e demais conteúdos sobre o tema surge uma questão importante: Como a padronização de medicamentos pode melhorar a assistência farmacêutica hospitalar e reduzir os custos dos medicamentos? Para resolver essa questão é necessário conhecimentos sobre assistência farmacêutica no âmbito hospitalar e sobre o processo de padronização de medicamentos.

Entende-se por assistência farmacêutica (AF) o conjunto de ações e de serviços que visam assegurar a assistência terapêutica integral e a promoção, a proteção e a recuperação da saúde nos estabelecimentos públicos e privados que desempenhem atividades farmacêuticas, tendo o medicamento como insumo essencial e visando ao seu acesso e ao seu uso racional (Brasil, 2014). O ciclo da assistência farmacêutica é composto por seleção, programação, aquisição, armazenamento, distribuição e dispensação de medicamentos, esse ciclo é fundamental para a gestão do medicamento pois possui etapas que garantem uma melhor qualidade dos medicamentos que são utilizados.

A padronização de medicamentos (PM) pode ser definida como a elaboração de uma lista de medicamentos fundamentais que devem compor os estoques da farmácia hospitalar, essa lista, por sua vez, facilita a aquisição de medicamentos pois são comprados apenas os medicamentos presentes na lista de padronizados. Uma padronização feita sobre critérios técnico-científicos pode ajudar a diminuir o número de medicamentos no estoque priorizando apenas medicamentos da lista de padronizados e consequentemente evitar problemas com medicamentos vencidos no estoque da farmácia. A lista de medicamentos padronizados deve ser divulgada para todos os setores do hospital para que os demais profissionais, técnicos e funcionários da instituição tenham ciência dos medicamentos que serão utilizados. Nesse contexto, esse este artigo tem como objetivo discutir o impacto da padronização de medicamentos na assistência farmacêutica hospitalar e nos custos dos medicamentos.

\section{Farmácia hospitalar}

A FH é a unidade clínico-assistencial, técnica e administrativa, onde se processam as atividades relacionadas à assistência farmacêutica, dirigida exclusivamente por farmacêutico, compondo a estrutura organizacional do hospital e integrada funcionalmente com as demais unidades administrativas e de assistência ao paciente (Brasil, 2010). A finalidade é garantir o uso racional de medicamentos que serão prescritos pelos médicos, para isso é necessário realizar um excelente planejamento na aquisição de fármacos e materiais hospitalares para preencher às necessidades e a demanda dos indivíduos hospitalizados, na mesma dimensão da sua utilização (Viana \& Nunes, 2019).

Por outro lado, a FH também contribui no processo de cuidado à saúde, prestando assistência ao paciente com qualidade, objetivando o uso seguro e racional de medicamentos, conforme preconiza a Política Nacional de Medicamentos, regulamentada pela Portaria n 3.916/98, do Ministério da Saúde (Sehnorin, 2017). 
A farmácia hospitalar possui vários objetivos, porém é preciso observar o alcance dos mesmos de forma consciente e apropriada. O modo como os objetivos são alcançados e a seriedade no cumprimento deles é o que mais importa. A farmácia propriamente dita e o farmacêutico hospitalar estão diretamente envolvidos nesses objetivos, são eles (Maia Neto, 2016): Planejamento, aquisição, análise, armazenamento, distribuição e controle de medicamentos e correlatos; Desenvolvimento e manipulação de fórmulas magistrais e oficinais; Produção de medicamentos e correlatos; Desenvolvimento de pesquisas e trabalhos próprios ou em colaboração com outros profissionais; Desenvolvimento de atividades didáticas; Adequar-se a problemas políticos, sociais, econômicos, financeiros e culturais do hospital; Estimular a implantação e o desenvolvimento da farmácia clínica.

Quanto a localização, instalação e arquitetura, a farmácia hospitalar deve manter equidistância dos demais setores do hospital pelo seguintes fatores: facilitar a distribuição de medicamentos e materiais médico-hospitalares; aprimorar o recebimento de produtos; facilitar o inter-relacionamento profissional; aumentar o poder de vigilância dos produtos em circulação no hospital; tornar mais fácil as vias de acesso para pacientes de ambulatório, principalmente no que diz respeito às farmácias satélite, destinadas a atendê-los; convívio entre o farmacêutico e o paciente passa a ser intenso, considerando a proximidade dos setores de atendimento à saúde. A aquisição de equipamentos deve ser feita de acordo com a necessidades do serviço e do hospital, a área total da farmácia deve ser proporcional ao tipo de trabalho e serviços a serem executados (Maia Neto, 2016).

A farmácia hospitalar compreende um local integrado as demais unidades assistenciais do hospital, sendo de extrema importância no que diz respeito ao uso seguro e racional de medicamentos, serviços e outros produtos de saúde. E isto é obtido por meio da assistência e atenção farmacêutica, que vai desde a seleção, aquisição, armazenamento, dispensação do medicamento, além de serviços especializados oferecidos, tais como, farmacovigilância, farmácia clínica, entre outros (Pelntir; Deuschle \& Deuschle, 2015).

Entende-se por assistência farmacêutica o conjunto de ações e de serviços que visem a assegurar a assistência terapêutica integral e a promoção, a proteção e a recuperação da saúde nos estabelecimentos públicos e privados que desempenhem atividades farmacêuticas, tendo o medicamento como insumo essencial e visando ao seu acesso e ao seu uso racional (Brasil, 2014). A assistência farmacêutica é um processo dinâmico e multidisciplinar, criado para abastecer os serviços de saúde com medicamentos de qualidade, disponibilizando aos pacientes acesso aos medicamentos que eles precisam. O farmacêutico é um profissional de saúde indispensável na garantia do acesso a medicamentos e seu uso seguro e racional, lembrando que o profissional qualificado é a garantia desse acesso e de uma assistência farmacêutica de qualidade (Pelentir; Deuschle \& Deuschle, 2015).

A Assistência Farmacêutica, no contexto hospitalar, engloba atividades relacionadas à logística, manipulação, controle de qualidade, atenção farmacêutica e farmácia clínica, conforme representado na Figura 1. Além disso, existem atividades intersetoriais, que requerem interação com outros setores do hospital (Brasil, 2019). 
Figura 1 - Esquema de assistência farmacêutica no âmbito hospitalar.

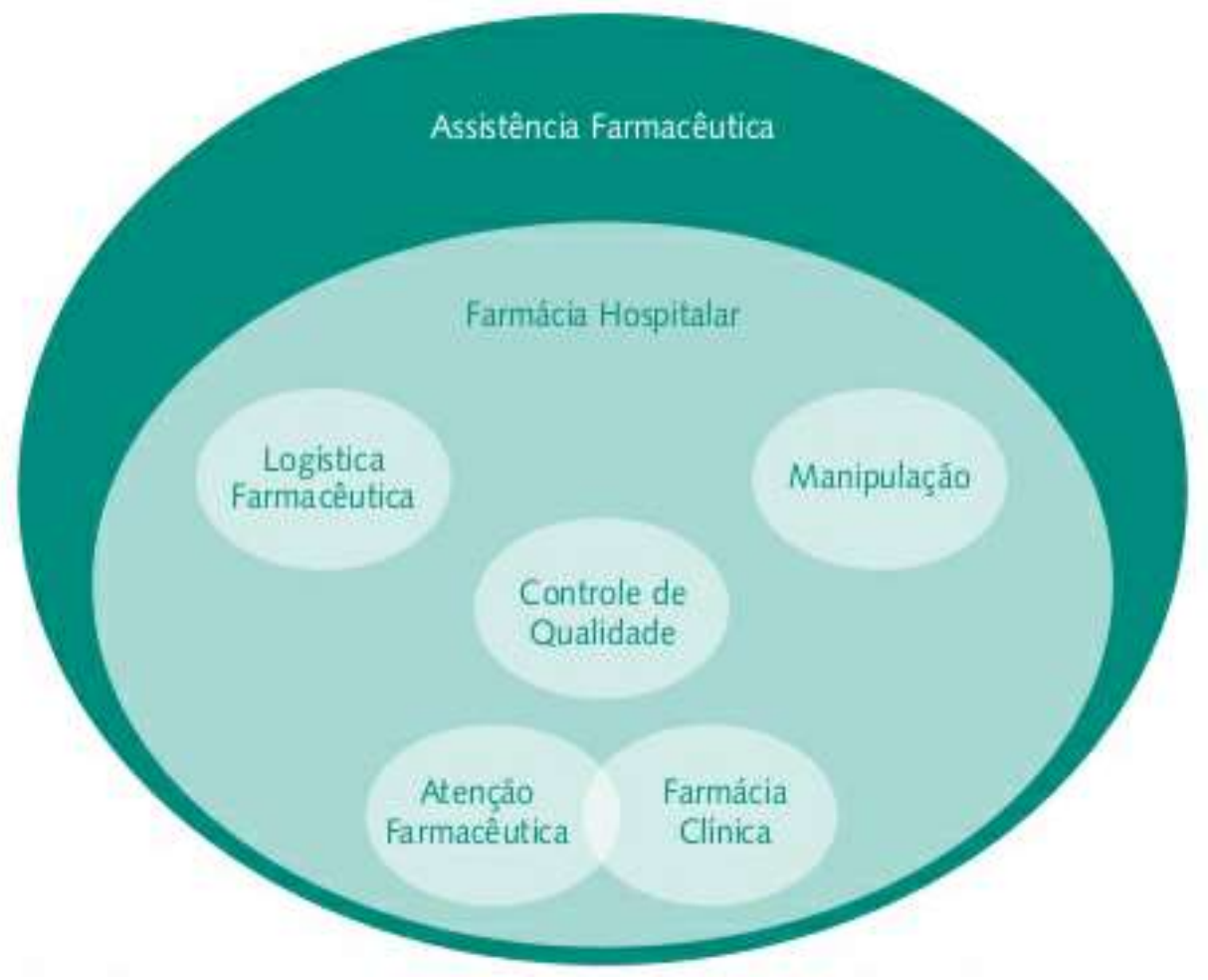

Fonte: Brasil (2017).

No âmbito da assistência farmacêutica, a compra de medicamentos é uma das principais ações, uma vez que, são fundamentais para o suporte às ações de saúde. Na área hospitalar, a política do uso racional de medicamentos deve ser inserida através de ações de padronização de fármacos. Esse processo de triagem deve obedecer a uma redução de custos e uso racional desses medicamentos (Silva, 2020).

\section{Comissão de farmácia e terapêutica}

A Comissão de Farmácia e Terapêutica (CFT) é muito importante para que a gestão da saúde seja realizada com maior segurança, qualidade e efetividade. De acordo com o Ministério da Saúde, a CFT pode ser definida como uma instância colegiada, de natureza técnico-científica, permanente, de caráter consultivo e deliberativo, que tem por finalidade assessorar o gestor e a equipe de saúde em assuntos referentes a medicamentos, visando garantir o uso seguro e racional. A CFT tem por finalidade assessorar a governança do hospital e auxiliar na consolidação de todas as políticas e práticas de utilização de medicamentos e outros produtos farmacêuticos, por meio da seleção e padronização de medicamentos, do estabelecimento de critérios para seu uso e avaliação dos itens selecionados (Brasil, 2019).

A CFT deve ser composta por membros de distintas áreas de atuação trabalhando em sintonia e realizando encontros periódicos, sendo capaz de elaborar e implementar intervenções para melhorar o uso de medicamentos e como consequência a saúde geral da população ao menor custo possível (Brandão, 2019).

A CFT tem como objetivos: Desenvolver Políticas relacionadas a avaliação, seleção e ao uso dos medicamentos; Apoiar o processo de aquisição de medicamentos e insumos farmacêuticos; Auxiliar na elaboração de programas de capacitação relacionados à utilização de medicamentos no âmbito institucional; Promover o uso seguro e racional de medicamentos, baseando-se em critérios preconizados pela OMS/OPAS/ANVISA (Brasil, 2019). 
A CFT tem como funções (Brandão, 2019): Seleção e padronização de medicamentos; Elaboração e atualização do guia farmacoterapêutico; Definição de diretrizes para uso racional de medicamentos; Elaboração de normas para prescrição, dispensação e uso de medicamentos; Avaliação para incorporação de novas tecnologias; Promoção e elaboração de protocolos clínicos de tratamento; Realizar estudos epidemiológicos da população e descrever o perfil farmacoepidemiológico e o impacto econômico dos medicamentos nas instituições de saúde; Desenvolver atividades de farmacovigilância e de risco, com monitoramento de reações adversas, queixa técnica e erros de medicação.

A CFT é formada por uma equipe multidisciplinar de farmacêuticos, médicos e várias especialidades atuantes em hospitais, um enfermeiro e um representante administrativo. A CFT é composta por (Nicolau, 2015): 1 Farmacêutico chefe da farmácia; 1 médico representante da clínica médica; 1 médico representante da clínica cirúrgica; 1 médico representante da pediatria; 1 médico presidente da CCIH e 1 enfermeiro chefe da equipe de enfermagem;

Dentre as diversas atribuições do farmacêutico na CFT, destaca-se a (Brasil, 2015): Atuar na escolha, análise crítica e utilização de estudos científicos que fundamentem a adequada seleção de medicamentos e produtos para saúde; Participar da elaboração de diretrizes clínicas e protocolos terapêuticos, observando normativas do Ministério da Saúde; Avaliar e estabelecer critérios para prescrição e uso de medicamentos e produtos para saúde não selecionados, eventualmente prescritos; Utilizar técnicas de farmacoeconomia para a avaliação dos medicamentos e outros produtos para saúde; Participar da definição de critérios que disciplinem a divulgação de medicamentos e produtos para saúde no ambiente hospitalar; Garantir a divulgação permanente da relação de medicamentos selecionados e dos produtos para saúde, destacando sempre as atualizações da relação promovidas pela Comissão; Utilizar indicadores epidemiológicos como critério do processo decisório de seleção; Zelar pela adesão e cumprimento da seleção de medicamentos e produtos para saúde e; Participar da elaboração do guia farmacoterapêutico. Parágrafo único - O farmacêutico poderá ocupar as funções de membro efetivo, secretário ou presidente da Comissão e, consequentemente, suas atribuições irão variar de acordo com o cargo ocupado.

\section{Padronização de medicamentos}

A necessidade de se estabelecer critérios para tratamento de enfermidades sempre levou os conhecedores da matéria e cuidadores de doentes a relacionarem remédios já existentes, mesmo que fossem utilizados de uma forma bem diferente do que hoje se conhece como medicamento. O papiro de Ebers talvez seja o documento mais antigo que remete ao conceito de padronização, pois ele continha centenas de fórmulas, indicação terapêutica e formas de preparo, era uma "padronização de drogas" uma vez que eram utilizadas partes de plantas que continham princípios ativos, o que indicava a necessidade de uniformização da terapêutica que prevalece até hoje, porém utilizando outros dados como o emprego de doses seguras e eficazes (Maia Neto, 2016).

A Padronização de Medicamentos (PM) pode ser entendida como uma relação de medicamentos básicos que devem constituir os estoques das farmácias hospitalares, visando atender os setores do hospital de acordo com suas necessidades e peculiaridades locais acarretando o uso racional de medicamentos. A análise das peculiaridades de cada instituição no processo de PM é de fundamental importância, pois cada hospital é uma instituição particular, com seus perfis e equipes. A padronização visa a diminuição de gastos de aquisição dos fármacos, facilitação das compras, armazenagem, manuseio e redução dos custos de produção manutenção dos produtos em estoque, proporcionando benefícios ao hospital como um todo (Viana \& Nunes, 2019).

Além disso a padronização de medicamentos pode sofrer influência de vários fatores tais como a síntese de novos fármacos, a existência de uma comissão que regulamenta a inclusão e exclusão de medicamentos e a política de medicamentos do ministério da saúde (Maia Neto, 2016). 
A Organização Mundial de Saúde (OMS) estabelece e disponibiliza a Relação Nacional de Medicamentos Essenciais (RENAME) com os medicamentos que devem atender as necessidades de saúde prioritárias da população. Assim, padronização de medicamentos deve correlacionar com critérios propostos pelo Ministério da Saúde (Nicolau, 2015).

A padronização de medicamentos tem como objetivos principais (Sampaio, 2016): Otimização do atendimento ao paciente por meio da seleção racional, aumento da qualidade da farmacoterapia; Facilitar a vigilância farmacológica, garantir segurança na prescrição e dispensação dos medicamentos e diminuir os índices de erros de medicação; Disciplinar o receituário e uniformizar a terapêutica para estabelecimento de protocolos e critérios; Redução dos custos da terapêutica e do estoque de medicamentos que apresentam mesmo fim terapêutico; Diminuição da quantidade de produtos em estoque e redução de fórmulas e formas farmacêuticas; redução no custo da aquisição de medicamentos e de manutenção do estoque; Agilidade na prescrição médica e no serviço de enfermagem; Melhorar a comunicação entre farmácia, equipe médica, pessoal de enfermagem e seções administrativas, assim como a simplificar as rotinas de aquisição, armazenamento, dispensação e controle.

A PM se divide em três tipos (Sampaio, 2016):

Padronização aberta: mesmo com listagem pré-definida, há fornecimento de qualquer medicamento, estando ou não incluído na lista. A listagem serve para orientar os prescritores. Permite-se a uso de outros medicamentos, desde que reembolsados pelo provador.

Padronização fechada: permite maior controle das prescrições e levantamento de necessidade de fármacos frequentemente solicitados. Medicamentos não padronizados dependem de solicitação especial para aquisição.

Padronização seletiva/parcialmente fechada: é um tipo de padronização aberta, onde à negativa de aquisição de medicamentos para propósitos específicos (cosméticos, perda de peso). Além disso a restrições na aquisição, apenas certas especialidades são habilitadas a prescrever certos fármacos, geralmente muito caros, ou que exigem acompanhamento especial.

A PM exige, para sua implantação, selecionar fármacos comprovadamente eficazes, e entre eles, definir aquele com melhor posologia e menor toxicidade, padronizar fármacos com menor valor de tratamento por dia e que sejam encontrados nas empresas em forma unitária, selecionar formas farmacêuticas adequadas para a dose e que possibilitem o fracionamento e substâncias farmacologicamente distintas (Viana \& Nunes, 2019).

A implantação da PM deve seguir quatro etapas (Sampaio, 2016): Estudo da estrutura técnica, administrativa e organizacional do hospital e a análise da regulamentação relacionada aos medicamentos; Formação da Comissão de Farmácia e Terapêutica (CFT); fazer levantamento dos fármacos utilizados no hospital que apresentam consumo médio contínuo e podem ser incluídos em métodos de aquisição. Em seguida, devem ser analisadas normas, protocolos e considerações econômicas; fazer a listagem com a seleção dos fármacos essenciais para arsenal terapêutico do hospital.

O processo de implantação da PM deve seguir os seguintes passos (Viana \& Nunes, 2019): Classificar os fármacos por Classe Terapêutica ou Grupos Farmacológico ou outro procedimento conveniente para o Hospital, com a utilização de uma demonstração simples e de fácil consulta; Requisitar a comissão do hospital o aconselhamento dos fármacos a serem englobados na relação de medicamentos padronizados; Optar pela Denominação Comum Brasileira - DCB ou na ausência desta escolher a Denominação Comum Internacional - DCI; Determinar os fármacos que serão incluídos na relação, retirando os medicamentos análogos (com exceção daqueles que possuir justificativa técnica); Divulgar a relação de Fármacos Padronizados por meio da Diretoria Clínica.

A curva $\mathrm{ABC}$ é uma ferramenta gerencial empregada para determinar a política de vendas, administração de estoques, programação da produção, estabelecimento de prioridades e outros problemas comuns nas empresas, ela é utilizada para separar os produtos de maior impacto, os quais geralmente estão em menor quantidade para se estabelecer critérios de gestão adequada à importância de cada fármaco sobre o valor total dos estoques (Viana \& Nunes, 2019). 
As classificações da curva ABC são definidas na seguinte forma (Ferranti; Santos \& Kranz, 2015):

Classe A: itens de maior importância financeira. São itens que merecem maior atenção na gestão, com acompanhamento diário de utilização e menor tempo de ressuprimento, pois possuem um alto valor agregado.

Classe B: Grupo intermediário, são financeiramente importantes também. Exigem um controle preciso não tão rigoroso quanto os da classe $\mathrm{A}$.

Classe C: Itens com menor importância. Exigem acompanhamento com menor prioridade.

Entre os objetivos da curva $\mathrm{ABC}$ destaca-se o: tratamento especial para itens da curva A; estratégias para controle de estoque conforme valor agregado; utilizar dados de controle da curva A como indicadores; Liquidez ao aumentar giro de determinados itens (Ferranti; Santos \& Kranz, 2015).

Para se obter um controle físico efetivo dos itens é necessário que os itens de classe A sejam mais frequentemente monitorados para verificar as quantidades e valores reais. Os itens de classe $\mathrm{C}$ exigem menos monitoramento, podendo serem monitorados uma vez por mês dependendo da empresa. Já os itens de classe B terão critérios de nível intermediário de monitoramento de estoque (Hümber \& Heinrichs, 2012).

Para inclusão ou exclusão na padronização, a farmácia deve ser chamada a apresentar monografias sobre os produtos, juntamente com uma descrição dos efeitos das mudanças propostas em relação à qualidade, custos e eficácia/ineficácia da terapia. Pode ser confeccionado um formulário para adição ou exclusão de medicamentos na padronização (Sampaio, 2016).

É de competência da CFT apresentar critérios para inclusão ou exclusão de medicamentos, mas para isso é necessário ter um documento formal com pelo menos as seguintes informações (Maia Neto, 2016): Identificação do hospital; Identificação da comissão; A denominação genérica do medicamento que deve ser incluído ou excluído, com as respectivas formas farmacêuticas, dosagens, posologia e indicação; Justificativa da inclusão; Citação dos trabalhos científicos que o acompanham; Previsão do consumo para um período determinado; Data e assinatura do responsável pela solicitação, de preferência como o "visto" do responsável pela unidade solicitante: definir fluxograma de encaminhamento da documentação, elaborar lista de padronizados e definir como instrumento básico para prescrição médica, rever e atualizar a lista de padronizados, estabelecer periodicidade com a divulgação da lista atualizada, estudar medicamentos do ponto de vista clínico, bio-farmacocinético e químico, emitindo parecer técnico sobre sua eficácia, registrar dados farmacológicos e clínicos relativos a novos medicamentos ou agentes terapêuticos propostos para uso hospitalar, divulgar informações relativas a estudos clínicos dos medicamentos incluídos ou excluídos e servir como órgão assessor ao corpo clínico e à administração do hospital em assuntos relacionados a medicamentos.

São critérios para inclusão de medicamentos na padronização (Viana \& Nunes, 2019): Selecionar apenas medicamentos de valor terapêutico comprovado, optando por fármacos de baixa toxicidade e aceitos pela ANVISA; Evitar diversidade de princípios ativos para a mesma utilização terapêutica; Padronizar fármacos cujos fornecedores foram avaliados tecnicamente pelo grupo interno de credenciamento; Optar por especialidades que não sejam combinações ou associações medicamentosas, priorizando às monodrogas; Escolher fármacos com baixo custo de aquisição, acondicionamento, dispensação e controle, sempre enfatizando a qualidade; Preferir a designação genérica do princípio ativo existente na lista de PM; Padronizar fármacos em dosagem, formas farmacêuticas e apresentação levando em conta a comodidade de administração, clareza para cálculo de dose ou fracionamento e optar por fármacos em que o valor da terapêutica por dia seja menor, garantindo a qualidade prioritariamente.

Já os critérios para exclusão de medicamentos na padronização são esses (Paula, 2016): Os medicamentos que possuírem comercialização não permitida ou interrompida através do órgão competente; Medicamentos que poderão ser trocados com vantagens, quando da inserção de diferente fármaco; Utilização em período estimado, que não justifique a padronização. (fonte: TNR 10 - justificado - espaço 1,5). 


\section{Metodologia}

Trata-se de uma pesquisa de campo de caráter descritivo e exploratório e de abordagem qualitativa que foi realizada na farmácia hospitalar do hospital regional de Conceição do Araguaia (HRCA) na qual houve uma entrevista direcionada ao farmacêutico responsável técnico utilizando um questionário com questões que visam alcançar os objetivos deste trabalho.

A pesquisa foi realizada na farmácia do Hospital Regional de Conceição do Araguaia (HRCA), que presta uma assistência em tempo integral (24 horas), atendendo e reabastecendo dentre, principalmente o setor de triagem/acolhimento, urgência e emergência, clínica médica, clínica cirúrgica, clínica ortopédica, clínica obstétrica, centro cirúrgico, Unidades de Terapia Intensiva (UTI) e Unidade de Cuidados Intermediários (UCI), para atender essa demanda a farmácia tem aproximadamente 200 itens, entre medicamentos e produtos para saúde, esses itens são divididos entre a farmácia central e as três farmácia s satélite.

A coleta de dados foi realizada por meio de uma entrevista, onde foi utilizado um questionário contendo 10 questões discursivas referentes ao tema da pesquisa, as 3 primeiras questões foram sobre o tipo de padronização de medicamentos utilizada no hospital, o processo de padronização e os critérios utilizados, já as demais 7 questões foram todas voltadas para discutir o impacto da padronização de medicamentos na assistência farmacêutica hospitalar e nos custos dos medicamentos, onde questionou-se o impacto da padronização em etapas do ciclo da assistência farmacêutica dentro do hospital bem como o seu impacto na elaboração de guias e diretrizes.

\section{Resultados e Discussão}

As respostas dadas pelo farmacêutico na entrevista foram confrontadas com dados encontrados na literatura disponível sobre o tema, para isso foram utilizados dados de artigos publicados nos últimos 5 anos, isto é, do ano de 2017 a 2021.

\section{Entrevista}

Questão 01. A padronização de medicamentos é o desenvolvimento de uma relação de medicamentos fundamentais que constituem os estoques da farmácia e que pode ser classificada em três tipos, padronização aberta, fechada e seletiva/parcialmente fechada. Sendo assim, qual o tipo/método de padronização de medicamentos utilizado pelo hospital?

Resposta: Seletiva/parcialmente fechada. Atualmente houve uma flexibilização maior na aquisição de medicamentos em decorrência das possibilidades de tratamento sem muito embasamento científico estando mais aberto ao corpo clínico no que diz respeito a inclusão/exclusão de meros itens.

As características assistenciais de cada hospital irão determinar qual tipo de padronização que será instituído. Um Hospital de urgência e emergência, por exemplo, atende vários tipos e pacientes, com as mais variadas enfermidades e condições clínicas. Logo, é coerente compreender as alternativas de tratamento para diversos quadros clínicos e a inclusão de fármacos específicos, como antídotos envenenamentos ou intoxicação (Silva, 2020). A padronização seletiva/parcialmente fechada é um tipo de padronização aberta onde à aquisição de fármacos para objetivos específicos (Viana \& Nunes, 2019) .

Questão 02. Para que haja padronização de medicamentos é necessário a formação de uma comissão de farmácia e terapêutica responsável pela seleção e padronização de medicamentos, elaboração e atualização de guias, diretrizes, normas, protocolos dentre outras atividades relacionadas a medicamentos. Sabendo disso, como ocorre a formação da comissão de farmácia e terapêutica (CFT) dentro do hospital?

Resposta: O ideal para se formar a comissão de farmácia e terapêutica dentro do hospital seria um farmacêutico presidindo a comissão, médicos especialistas de cada clínica (UCE, UCI neonatal, UTI...), um representante da equipe de enfermagem, um representante da CCIH, um representante do setor de compras, instituída e publicada em diário oficial. Mas. 
Entretanto, atualmente, uma comissão não foi instituída. Atualmente, o que se vê na realidade é um esforço anual da farmácia por meio da via documental e contato com a equipe médica solicitando sugestões de inclusãolexclusão de medicamentos com justificativa e referência para tal, logo, nem todos dão o retorno.

A CFT é uma instância multiprofissional, deliberativa, consultiva e educativa, localizada dentro de hospitais e outros serviços de saúde, responsável pela seleção dos fármacos para o arsenal terapêutico, por meio de um processo participativo, contínuo, dinâmico e multidisciplinar, visando garantir o acesso aos medicamentos, aderindo critérios de segurança, qualidade, eficácia e tendo autonomia para produzir, incluir e excluir fármacos do arsenal terapêutico (Viana \& Nunes, 2019). As CFTs não são obrigatórias, mas é recomendação da OMS que sejam instaladas como órgão responsável pela seleção e padronização de medicamentos. Em países desenvolvidos, essas comissões estão relacionadas com o sucesso no monitoramento e promoção do uso racional de medicamentos além da redução de gastos nos serviços de saúde, já nos países subdesenvolvidos, a institucionalização das CFTs é pouco comum, assim como os estudos de avaliação de impacto clínicos e econômicos que essas comissões podem gerar (Brandão, 2019).

Questão 03. Para uma padronização com medicamentos seguros e eficazes existem inúmeros critérios técnicocientíficos que devem ser rigorosamente seguidos, como a revisão de estudos clínicos sobre o medicamento e estudos farmacoeconômicos. Sabendo disso, quais os critérios utilizados pelo farmacêutico junto com a equipe profissional para a seleção e padronização de medicamentos no hospital?

Resposta: Selecionar o menor número de medicamentos possíveis que atendam o maior número de patologias (espectro amplo de ação), considerar o perfil de atendimento hospitalar, busca por medicamentos de baixo custo, considerar o corpo clínico atual, considerar evidências clínica e científicas, priorizar medicamentos com maior frequência de prescrição (maior uso), em caso de medicamentos de baixo uso, considerar exclusão.

A seleção de medicamentos tem como produto um rol de medicamentos fornecidos pelo hospital, se não à seleção, não a como definir o que deve ou o que não deve ser padronizado (Pereira; Macedo \& Solto, 2017). Quanto ao perfil de atendimento hospitalar, é fundamental que analise as peculiaridades de cada instituição no processo de PM pois cada hospital é uma instituição particular com seus perfis e equipes. Devem ser selecionados fármacos com eficácia clínica e definir, entre estes, aquele com maior comodidade patológica e quanto ao baixo custo, devem ser padronizados medicamentos com menor valor de tratamento por dia (Viana \& Nunes, 2019).

Questão 04. Um dos objetivos da padronização de medicamentos é a redução dos custos da terapêutica e do estoque de medicamentos que apresentam mesmo fim terapêutico. Sabendo disso, qual o impacto da padronização de medicamentos na aquisição de medicamentos, a padronização aumenta ou diminui os gastos e como seria o custo com aquisição de medicamentos caso não houvesse padronização?

Resposta: A padronização além de organizar, facilitar e agilizar o tratamento, reduz significativamente os custos com medicamentos, otimizando o recurso público. Em caso de não padronização, obviamente o custo com aquisição seria maior, pois não haveria uma lista de medicamentos padronizados com suas respectivas quantidades para seguir.

Os medicamentos são caros, sendo responsáveis por 30\% a 50\% dos orçamentos de saúde, e, num típico sistema de abastecimento, até $70 \%$ do financiamento investido em medicamentos essenciais pode ser perdido ou desperdiçado, o que faz sua gestão ser bastante diferente de outros produtos de consumo (Andrade, 2017). Os custos dos medicamentos devem ser considerados mediante ao processo de administração dos medicamentos e a correta análise do custo total do tratamento $\mathrm{x}$ período de internação é primordial para escolha dos medicamentos (Silva, 2020).

Questão 05. A padronização de medicamentos consiste na elaboração de uma lista de medicamentos fundamentais que devem compor os estoques da farmácia, essa lista, por sua vez, facilita a aquisição de medicamentos e a gestão do estoque, no entanto existem outras atividades importantes como vigilância farmacológica, prescrição de medicamentos seguros e 
eficazes e elaboração de protocolos de tratamento. Sendo assim, qual o impacto da padronização de medicamentos na vigilância farmacológica/farmacovigilância hospitalar, a padronização facilita ou não esse serviço?

Resposta: Facilita um processo investigativo, uma vez que os questionários de farmacovigilância podem ser elaborados de acordo com os medicamentos padronizados.

Farmacovigilância é definida como "ciência e atividade relativa à identificação, avaliação, compreensão e prevenção de efeitos adversos ou qualquer problema possível relacionado com fármacos" e é a responsável pelo acompanhamento do desempenho dos medicamentos disponíveis no mercado. Um aspecto negativo relacionado às reações adversas a medicamentos é o aumento dos gastos hospitalares, com valores que variam de 5,3\% a 8,5\% no período de internação, onerando desnecessariamente as instituições de saúde (Côrrea; Lorenz \& Colet, 2017).

Questão 06. A padronização de medicamentos é vista como uma forma de facilitar a gestão do estoque de medicamentos, um de seus objetivos é a diminuição da quantidade de produtos em estoque e redução de fórmulas e formas farmacêuticas. Sabendo disso qual o impacto real da padronização de medicamentos na gestão do estoque dos medicamentos, ela aumenta ou reduz a quantidade de produtos no estoque, ela simplifica ou complica a gestão do estoque de medicamentos da farmácia?

Resposta: Impacta positivamente, reduz a quantidade em estoque e simplifica a gestão do estoque de medicamentos.

A padronização é uma das soluções mais viáveis para definir o que se deve manter em estoques (Lima; Sousa; Oliveira \& 2020), ela oferece inúmeras vantagens, dentre elas a confiança e conforto dos profissionais de saúde pela convicção que os medicamentos e correlatos necessários para a prestação de serviço estarão disponíveis e em quantidade adequada para o atendimento (De Lima; De Castro \& Fernandes, 2019).

Questão 07. O uso de medicamentos pelos profissionais e de saúde e pelos pacientes no hospital para tratar determinadas doenças exige cuidados e critérios que podem ser estabelecidos em protocolos de tratamento e/ou guias farmacoterapêuticos. Sabendo disso qual o impacto da padronização de medicamentos na elaboração de protocolos e guias para o uso correto de medicamentos?

Resposta: A padronização facilita a criação de protocolos clínicos de uso dos medicamentos, facilitando a criação de critérios de escolha na sua utilização, minimizando erros e otimizando a terapia.

A padronização de um medicamento baseia-se na análise das melhores evidências clínicas disponíveis e esse processo é fundamental para a disponibilização de medicamentos eficazes, seguros e que mantenham a sustentabilidade do sistema de saúde (Silva; De Almeida \& Pessoa, 2017). Após a padronização é importante que o hospital tenha um Guia Farmacoterapêutico desenvolvido pela CFT. Este guia contém orientações posológicas e terapêuticas básicas visando fornecer ao médico uma ferramenta de consulta rápida. Além disso, é de suma importância o desenvolvimento de boletins, manuais de orientação e protocolos clínicos, que possuam orientações de segurança de utilização de todos os medicamentos padronizados num hospital. O desenvolvimento de protocolos envolve segurança na prescrição, uso e administração de medicamentos e deve ser aplicado em todos os estabelecimentos que prestam cuidados à saúde (Silva, 2017).

Questão 08. A dispensação de medicamentos é o ato de distribuir um ou mais medicamentos ao paciente, geralmente com apresentação de uma receita médica. O farmacêutico, na farmácia hospitalar é responsável por dispensar medicamentos para os demais setores do hospital. Para que ocorra uma boa dispensação no hospital o profissional deve tomar certos cuidados e seguir alguns critérios. Assim, como a padronização de medicamentos se encaixa no exercício de dispensação de medicamentos, qual o impacto?

Resposta: Neste caso a padronização só irá ajudar devido a seleção de medicamentos diminuir as possibilidades disponíveis. O que de fato atua na facilitação da dispensação é o sistema de distribuição adotado pelo hospital. 
As farmácias hospitalares têm por objetivo dispensar medicamentos sob a prescrição médica, na quantidade e especificações solicitadas, no prazo requisitado, oferecendo segurança e favorecendo o uso racional dos medicamentos, o profissional farmacêutico deve estabelecer um sistema de dispensação eficiente e seguro para os usuários, podendo ser considerado, um ponto importante para a racionalidade e adequação ao tratamento dos pacientes, e também no aspecto financeiro do hospital, antepondo-se com alguma determinação o funcionamento congruente ou não da farmácia e se os medicamentos recebidos pelos pacientes estão dentro dos critérios de qualidade e segurança (De Oliveira \& Garcia, 2020).

A dispensação de medicamentos na farmácia hospitalar é um dos meios de aproximar o serviço de farmácia á segurança do paciente, quanto mais eficiente o sistema de dispensação, menor será a incidência de erros e consequentemente, melhor será o serviço oferecido ao paciente (Makaruk \& Rissato, 2017).

Questão 09. Para tratar e prevenir certos problemas de saúde no hospital, como as infecções causadas por bactérias multirresistentes, é necessário o uso de medicamentos adequados, na dose adequada e no tempo adequado. Sendo assim, qual o impacto da padronização de medicamentos no tratamento e na prevenção de doenças e de vários outros problemas de saúde que ocorrem no hospital?

Resposta: Impacta positivamente, uma vez que são estabelecidos critérios para prescrição e escolha de antibioticoterapia, reduzindo a possibilidade de indução à resistência bacteriana, assim como a criação de superbactérias. Reduz a possibilidade de infecção hospitalar.

A padronização de fármacos possibilita a equipe multiprofissional procedimentos para avaliar e selecionar os medicamentos, os protocolos de orientação para utilização e a descrição das regras que colaboram para o sucesso do processo de utilização do fármaco (Viana \& Nunes, 2019). Além disso é importante que, baseado na lista de medicamentos padronizados de um hospital, haja um processo de educação farmacológica dos profissionais de saúde da instituição, induzindo reflexão crítica acerca da sua escolha e utilização (De Freitas, 2017).

Questão 10. Problemas relacionados aos medicamentos (PRMs) são quais quer eventos indesejáveis apresentados pelo paciente, que envolvam ou suspeita-se de estarem relacionados com a farmacoterapia. Esses problemas podem ocorrer em qualquer lugar, inclusive no hospital. Sabendo disso, qual o impacto da padronização de medicamentos na prevenção e resolução de problemas relacionados a medicamentos dentro do ambiente hospitalar?

Resposta: A padronização de medicamentos facilita na previsão de problemas relacionados a medicamentos que podem ocorrer, onde pode-se realizar uma planilha com os medicamentos padrões e já padronizar os possíveis problemas que podem surgir, facilitando a triagem e suas resoluções.

Os objetivos da padronização de medicamentos, nesse caso, são elevar a qualidade da farmacoterapia, garantir a segurança na prescrição e administração dos fármacos, regularizar a prescrição médica e padronizar a terapia (Viana \& Nunes, 2019). A padronização de medicamentos em um hospital é resultado da seleção, que, por sua vez, pressupõe a avaliação criteriosa e baseada em evidências, comparando-se todas as alternativas terapêuticas disponíveis para determinada doença ou situação clínica (Pereira, 2017).

\section{Considerações Finais}

A FH é um setor que demanda autos valores orçamentários fazendo com que pessoas responsáveis pela instituição adotem métodos que garantam maior controle sobre as contas do hospital. A CFT é uma junta multiprofissional responsável pela seleção, padronização e outras questões relacionadas a medicamentos e tem sua importância no processo de padronização de medicamentos. Apesar do HRCA não dispor de uma CFT para executar a padronização de medicamentos ainda assim é possível realizar essa atividade sem a necessidade de uma comissão. 
A padronização de medicamentos é uma atividade muito importante empregada pela equipe de profissionais do hospital para que haja um controle maior sobre os medicamentos disponíveis no estoque e que tem como ferramenta uma lista de medicamentos selecionados sobre critérios técnico-científicos e padronizados. A padronização é feita pelos profissionais sobre diferentes métodos e é aplicada na grande maioria dos hospitais.

A presença de uma lista de medicamentos padronizados facilita a aquisição uma vez que são adquiridos somente fármacos desta lista, isso não só torna fácil a aquisição como também reduz os seus custos já que os medicamentos selecionados são seguros, eficazes e custo-efetivos. Facilita o controle de estoque pois são adquiridos apenas quantidades específicas de medicamentos baseado na lista de padronizados. Facilita o processo de farmacovigilância uma vez que facilita a prevenção de reações adversas e, também, facilita a escolha do medicamento para tratamento de patologias de acordo com o perfil de atendimento da instituição.

\section{Referências}

Aizenstein, M. L., \& Tomassi, M. H. (2011). Problemas relacionados a medicamentos; reações adversas a medicamentos e erros de medicação: a necessidade de uma padronização nas definições e classificações. Revista de Ciências Farmacêuticas Básica e Aplicada, 32(2). https://rcfba.fcfar.unesp.br/index.php/ojs/article/view/341/339.

Andrade, N. N. D. (2017). Avaliação de economicidade da política pública de assistência farmacêutica básica: uma comparação dos custos dos medicamentos em Alagoas do Programa de Assistência Farmacêutica Básica e do Programa Farmácia Popular do Brasil. https://repositorio.ufba.br/ri/handle/ri/24538.

Brandão, C. G. S. (2019). Análise da comissão de farmácia e terapêutica do município de Teutônia (RS) nos anos de 2014 a 2017. https://www.lume.ufrgs.br/handle/10183/201709

Brasil, Ministério da Saúde. Portaria $\mathrm{n}^{\circ}$ 4.283, de 30 de dezembro de (2010). Aprova as diretrizes e estratégias para organização, fortalecimento e aprimoramento das ações e serviços de farmácia no âmbito dos hospitais. http://bvsms.saude.gov.br/bvs/saudelegis/gm/2010/prt4283_30_12_2010.html..

Brasil. Conselho Federal de Farmácia. Resolução n ${ }^{\circ} 619$ de 27 de novembro de (2015). Dá nova redação aos artigos $1^{\circ}$ e $2^{\circ}$ da Resolução/CFF nº 449 de 24 de outubro de 2006, que dispõe sobre as atribuições do Farmacêutico na Comissão de Farmácia e Terapêutica. https://pesquisa.in.gov.br/imprensa/jsp/visualiza/index.jsp?data=07/12/2015\&jornal=1\&pagina=115\&totalArquivos=120.

Brasil. Conselho Regional de Farmácia do Estado de São Paulo. Farmácia Hospitalar. São Paulo, (2019). http://www.crfsp.org.br/index .php?option=com_content $\&$ view=article \&id=8248-cartilha-de-farmacia-hospitalar.html .

Brasil. Lei $\mathrm{n}^{\circ}$ 13.021, de agosto de (2014). Dispõe sobre o exercício e fiscalização das atividades farmacêuticas.http://www.planalto.gov.br/ ccivil_03/_ato2011-2014/2014/lei/113021.htm .

Brasil. Ministério da educação. Diretriz para Constituição e Operacionalização das Comissões de Farmácia e Terapêutica. 5. ed. (português), Brasília: EBSERH, (2018). http://www2.ebserh.gov.br/documents/695105/2749071/SEI_SEDE+-+0251751+-+DAS+-+Manual+de+CFT+Fasc\%C3\%ADculo+I_1\% C2\%AA+ed.LINK.pdf/fb2ab8fd-9b07-4572-963d-fc58a738d791.

Côrrea, J., Lorenz, C \& de Fátima Colet, C. (2017). Comparação entre estratégias de farmacovigilância hospitalar nos países da américa latina. Revista Contexto \& Saúde, 17(33), 155-166. file://C:/Users/guilh/Downloads/2970-Texto\%20do\%20artigo_-33051-1-10-20171107.pdf.

Freitas, E. L. D. Proposta de manejo de medicamentos não padronizados trazidos por pacientes em um hospital público especializado em cardiologia do município do Rio de Janeiro. https://app.uff.br/riuff/handle/1/15615

De Lima, F. A. C., de Castro, S. F., Fernades, W. S., de Lima, L. B \& de Lapena, S. A. B. (2019). Estratégias de organização de medicamentos e correlatos numa farmácia satélite no centro cirúrgico de um hospital filantrópico de Jacareí/SP. Brazilian Journal of Health Review, 2(4), 2699-2708. https://www.brazilianjournals.com/index.php/BJHR/article/view/1975

De Oliveira, S. M. M \& Garcia, m. A. T (2020). Gestão de processos de dispensação de medicamentos em unidades hospitalares. http://fait.revista.inf.br/imagens_arquivos/arquivos_destaque/pUeAMPYj5CV6Vh6_2021-3-9-15-39-55.pdf

Ferranti, E; Santos, C. S \& Kranz, L. F. (2015). Gestão de estoque de medicamentos utilizando classificação ABC em um hospital público. https://www.lume.ufrgs.br/handle/10183/130297

Humber, E. S \& Heinrichs, A. (2012). Utilização da curva abc para dimensionamento do estoque de medicamentos e materiais hospitalares, em unidade pública de saúde no município de Santa Cruz do Sul-RS. https://silo.tips/download/palavras-chave-curva-abc-classificaao-de-estoque-rotatividade

Lima, J. V. O.; Souza, R. G. C.; Oliveira, B. E. S.; Souza, C. F. A. J.; Silva, A. R.; Silva, I. P.; Barbosa, M. C. N. A.; Lopes, L. A. S.; Mendes, F. M. A.; Santana, L. S. O. S \& Júnior, J, L, P. (2020). Avaliação Farmacêutica da relação de Medicamentos padronizados em um Hospital psiquiátrico de referência. Revista de Casos e Consultoria, 11(1) https://periodicos.ufrn.br/casoseconsultoria/article/view/20766.

de Oliveira Lima, J. V., Sousa, R. G. C., da Silva Oliveira, B. E., de Jesus Sousa, C. F. A., da Silva, A. R., Silva, I. P \& Júnior, J. L. P. (2020). Avaliação Farmacêutica da relação de Medicamentos padronizados em um Hospital psiquiatrico de referência. Revista de Casos e Consultoria, 11(1), e1119-e1119. https://periodicos.ufrn.br/casoseconsultoria/article/view/20766 
Research, Society and Development, v. 10, n. 14, e112101421201, 2021

(CC BY 4.0) | ISSN 2525-3409 | DOI: http://dx.doi.org/10.33448/rsd-v10i14.21201

Maia Neto, J. F. M. (2016). Farmácia Hospitalar e suas interfaces com a saúde. Rx Editora e Publicidade. https://books.google.com.br/books?hl=ptBR\&lr=\&id=XutS_BRhomoC\&oi=fnd\&pg=PA5\&dq=farm\%C3\%A1 cia+hospitalar+e+suas+interfaces+com+a+sa\%C3\%BAde\&ots=L5bOtvpl3M\&sig=Dtwi X4Vh5T32yTXp3fOp3ZmgK-4\#v=onepage\&q=farm\%C3\%A1cia\%20hospitalar\%20e\%20suas\%20interfaces\%20com\%20a\%20sa\%C3\%BAde\&f=false.

Makaruk, C. E \& Rissato, M. A. R (2018). Sistema de dispensação de medicamentos da farmácia inserida no ambiente hospitalar. https://bdm.ufmt.br/handle/1/1319.

Mendes, C. B. N. M., da Silva, S. D. S., \& de Cavalcanti, R. L. (2015). Intervenção De Excelência: Atuação Do Farmacêutico Na Padronização De Antimicrobianos Frente Às Comissões De Controle De Infecção Relacionada A Assistência À Saúde. Revista Presença, 1(3), 40-64. https://revistapresenca.celsolisboa.edu.br/index.php/numerohum/article/view/65

Lino, A. C. B., Nascimento, F. G., Vieira, D. C. R., \& Senhorin, G. Z. (2020). Gestão das solicitações de medicamentos não padronizados em um hospital universitário. VITTALLE-Revista de Ciências da Saúde, 32(1), 70-75. https://www.seer.furg.br/vittalle/article/view/8896

Nicolau, M. F. M. (2015). Estudo de solicitações de medicamentos não padronizados enviados a Farmácia do Hospital das Clínicas da Faculdade de Medicina de Ribeirão Preto (HCFMRP-USP). https://pesquisa.bvsalud.org/portal/resource/pt/ses-31796

Paula, L. B G. (2016). Importância da padronização de medicamentos na farmácia hospitalar. https://www.ccecursos.com.br/img/resumos/farmacia/tcc-larizanaiara-borba-gomes-de-paula.pdf

Pelentir, M., Deuschle, V. C. K. N., \& Deuschle, R. A. N. (2015). Importância da assistência e atenção farmacêutica no ambiente hospitalar. Ciência \& tecnologia, 1(1), 20-28. http://dx.doi.org/10.33053/cientec.v1i1.487

Pereira, P. A. R., De Macêdo, L. A; Souto, R. A. D. M (2017). Atualização da padronização de medicamentos e elaboração do guia farmacoterapêutico de um hospital Universitário. https://repositorio.ufpb.br/jspui/handle/123456789/3734

Sampaio, J. N. (2016). Padronização de medicamentos. http://docplayer.com.br/8353770-Padronizacao-de-medicamentos.html

Silva, E., de Almeida, K. C., \& Pessoa, G. S. C. (2017). Análise do gasto com judicialização de medicamentos no Distrito Federal, Brasil. Cadernos IberoAmericanos de Direito Sanitário, 6(1), 112-126. https://www.cadernos.prodisa.fiocruz.br/index.php/cadernos/article/view/358. 9 set. 2021

Silva, K. S. (2020). A importância da padronização de medicamentos em um hospital. https://monografias.ufrn.br/jspui/bitstream/1234567 89/11134/1/AImport\%c 3\%a2nciaDaPadroniz a\%c3\%a7\%c3\%a3oDeMedicamentosEmUmHospital_Silva_2020

Silva, M. D. R. Estudo de administração de medicamentos através de cateter de nutrição enteral em hospital federal do Rio de Janeiro. https://app.uff.br/riuff/handle/1/16574

Viana, A. M., \& Nunes, J. D. S. (2019). Padronização de medicamentos na farmácia hospitalar. http://repositorio.faema.edu.br/handle/123456789/2466 